\title{
UNIFORM LIPSCHITZ AND ASYMPTOTIC STABILITY FOR PERTURBED DIFFERENTIAL SYSTEMS
}

\author{
Yoon Hoe Goo* and Yinhua Cui**
}

\begin{abstract}
In this paper, we investigate uniform Lipschitz and asymptotic stability for perturbed differential systems using integral inequalities.
\end{abstract}

\section{Introduction}

Dannan and Elaydi [7] introduced a new notion of stability, which is called uniform Lipschitz stability (ULS), for systems of differential equations. For linear systems, the notions of uniform Lipschitz stability and that of uniform stability are equivalent. However, for nonlinear systems, the two notions are quite distinct. This notion of ULS lies somewhere between uniform stability on one side and the notions of asmptotic stability in variation of Brauer[3] and uniform stability in variation of Brauer and Strauss[2] on the other side. Also, Elaydi and Farran [8] introduced the notion of exponential asymptotic stability(EAS) which is a stronger notion than that of ULS. They studied the properties of EAS dynamical systems on a compact Riemannian manifold, and gave some analytic criteria for an autonomous differential system and its perturbed systems to be EAS. Gonzalez and Pinto[9] investigated the asymptotic behavior and boundedness of the solutions of nonlinear differential systems.

In this paper, we investigate uniform Lipschitz and asymptotic stability for solutions of the nonlinear differential systems using integral inequalities. The method incorporating integral inequalities takes an important place among the methods developed for the qualitative analysis of solutions to linear and nonlinear system of differential equations.

Received September 02, 2013; Accepted October 11, 2013.

2010 Mathematics Subject Classification: Primary 34D10.

Key words and phrases: uniformly Lipschitz stabe, uniformly Lipschitz stabe in variation, exponentially asymptotically stabe, exponentially asymptotically stabe in variation.

Correspondence should be addressed to Yoon Hoe Goo, yhgoo@hanseo.ac.kr. 
In the presence the method of integral inequalities is as efficient as the direct Lyapunov's method.

\section{Preliminaries}

We consider the nonlinear nonautonomous differential system

$$
x^{\prime}(t)=f(t, x(t)), \quad x\left(t_{0}\right)=x_{0},
$$

where $f \in C\left(\mathbb{R}^{+} \times \mathbb{R}^{n}, \mathbb{R}^{n}\right), \mathbb{R}^{+}=[0, \infty)$ and $\mathbb{R}^{n}$ is the Euclidean $n$ space. We assume that the Jacobian matrix $f_{x}=\partial f / \partial x$ exists and is continuous on $\mathbb{R}^{+} \times \mathbb{R}^{n}$ and $f(t, 0)=0$. Also, consider the perturbed differential system of (2.1)

$$
y^{\prime}=f(t, y)+\int_{t_{0}}^{t} g(s, y(s), T y(s)) d s, y\left(t_{0}\right)=y_{0},
$$

where $g \in C\left(\mathbb{R}^{+} \times \mathbb{R}^{n} \times \mathbb{R}^{n}, \mathbb{R}^{n}\right), g(t, 0,0)=0$ and $T$ is a continuous operator mapping from $C\left(\mathbb{R}^{+}, \mathbb{R}^{n}\right)$ into $C\left(\mathbb{R}^{+}, \mathbb{R}^{n}\right)$. The symbol $|\cdot|$ donotes arbitrary vector norm in $\mathbb{R}^{n}$.

In our subsequent discussion we assume that for any two continuous functions $u, v \in C(I)$ where $I$ is the closed interval, the operator $T$ satisfies the following property:

$$
u(t) \leq v(t), 0 \leq t \leq t_{1}, t_{1} \in I,
$$

imples $T u(t) \leq T v(t), 0 \leq t \leq t_{1}$, and $|T u| \leq T|u|$.

Let $x\left(t, t_{0}, x_{0}\right)$ be denoted the unique solution of $(2.1)$ through $\left(t_{0}, x_{0}\right)$ in $\mathbb{R}^{+} \times \mathbb{R}^{n}$ with $x\left(t_{0}, t_{0}, x_{0}\right)=x_{0}$, existing on $\left[t_{0}, \infty\right)$. Then we can consider the associated variational systems around the zero solution of (2.1) and around $x(t)$, respectively,

$$
v^{\prime}(t)=f_{x}(t, 0) v(t), v\left(t_{0}\right)=v_{0}
$$

and

$$
z^{\prime}(t)=f_{x}\left(t, x\left(t, t_{0}, x_{0}\right)\right) z(t), z\left(t_{0}\right)=z_{0} .
$$

The fundamental matrix $\Phi\left(t, t_{0}, x_{0}\right)$ of $(2.4)$ is given by

$$
\Phi\left(t, t_{0}, x_{0}\right)=\frac{\partial}{\partial x_{0}} x\left(t, t_{0}, x_{0}\right)
$$

and $\Phi\left(t, t_{0}, 0\right)$ is the fundamental matrix of $(2.3)$.

Before giving further details, we recall some notions of stability that we need in the sequel[8]. 
Definition 2.1. The system (2.1) (the zero solution $x=0$ of $(2.1)$ ) is called

(S) stable if for any $\epsilon>0$ and $t_{0} \geq 0$, there exists $\delta=\delta\left(t_{0}, \epsilon\right)>0$ such that if $\left|x_{0}\right|<\delta$, then $|x(t)|<\epsilon$ for all $t \geq t_{0} \geq 0$,

(US) uniformly stable if the $\delta$ in (S) is independent of the time $t_{0}$,

(ULS) uniformly Lipschitz stable if there exist $M>0$ and $\delta>0$ such that $|x(t)| \leq M\left|x_{0}\right|$ whenever $\left|x_{0}\right| \leq \delta$ and $t \geq t_{0} \geq 0$,

(ULSV) uniformly Lipschitz stable in variation if there exist $M>0$ and $\delta>0$ such that $\mid \Phi\left(t, t_{0}, x_{0}\right) \leq M$ for $\left|x_{0}\right| \leq \delta$ and $t \geq t_{0} \geq 0$,

(EAS) exponentially asymptotically stable if there exist constants $K>0$, $c>0$, and $\delta>0$ such that

$$
|x(t)| \leq K\left|x_{0}\right| e^{-c\left(t-t_{0}\right)}, 0 \leq t_{0} \leq t
$$

provided that $\left|x_{0}\right|<\infty$,

(EASV) exponentially asymptotically stable in variation if there exist constants $K>0$ and $c>0$ such that

$$
\left|\Phi\left(t, t_{0}, x_{0}\right)\right| \leq K e^{-c\left(t-t_{0}\right)}, 0 \leq t_{0} \leq t
$$

provided that $\left|x_{0}\right|<\infty$.

We give some related properties that we need in the sequel.

We need Alekseev formula to compare between the solutions of (2.1) and the solutions of perturbed nonlinear system

$$
y^{\prime}=f(t, y)+g(t, y), y\left(t_{0}\right)=y_{0},
$$

where $g \in C\left(\mathbb{R}^{+} \times \mathbb{R}^{n}, \mathbb{R}^{n}\right)$ and $g(t, 0)=0$. Let $y(t)=y\left(t, t_{0}, y_{0}\right)$ denote the solution of (2.5) passing through the point $\left(t_{0}, y_{0}\right)$ in $\mathbb{R}^{+} \times \mathbb{R}^{n}$.

The following is a generalization to nonlinear system of the variation of constants formula due to Alekseev [1].

LEMma 2.2. If $y_{0} \in \mathbb{R}^{n}$, then for all $t$ such that $x\left(t, t_{0}, y_{0}\right) \in \mathbb{R}^{n}$,

$$
y\left(t, t_{0}, y_{0}\right)=x\left(t, t_{0}, y_{0}\right)+\int_{t_{0}}^{t} \Phi(t, s, y(s)) g(s, y(s)) d s .
$$

Lemma 2.3. [6] Let $u, f, g, w \in C\left(\mathbb{R}^{+}\right), w(u)$ be nondecreasing in $u$ and $\frac{1}{v} w(u) \leq w\left(\frac{u}{v}\right)$ for some $v>0$. If ,for some $c>0$,

$u(t) \leq c+\int_{t_{0}}^{t} f(s) u(s) d s+\int_{t_{0}}^{t} f(s)\left\{\int_{t_{0}}^{s} g(\tau) w(u(\tau)) d \tau\right\} d s, \quad t \geq t_{0} \geq 0$,

then

$$
u(t) \leq W^{-1}\left[W(c)+\int_{t_{0}}^{t} g(s) d s\right] \exp \left(\int_{t_{0}}^{t} f(s) d s\right), t_{0} \leq t<b_{1},
$$


where $W(u)=\int_{u_{0}}^{u} \frac{d s}{w(s)}, u>0, u_{0}>0, W^{-1}(u)$ is the inverse of $W(u)$ and

$$
b_{1}=\sup \left\{t \geq t_{0}: W(c)+\int_{t_{0}}^{t} g(s) d s \in \operatorname{domW}^{-1}\right\} .
$$

Lemma 2.4. [12] Let $u, f, g, h \in C\left(\mathbb{R}^{+}\right), w \in C((0, \infty))$ and $w(u)$ be nondecreasing in $u, u \leq w(u)$. Suppose that for some $c>0$,

$u(t) \leq c+\int_{t_{0}}^{t} f(s) w(u(s)) d s+\int_{t_{0}}^{t} g(s)\left(\int_{t_{0}}^{s} h(\tau) u(\tau) d \tau\right) d s, \quad 0 \leq t_{0} \leq t$.

Then

$$
\left.u(t) \leq W^{-1}\left[W(c)+\int_{t_{0}}^{t}\left(f(s)+g(s) \int_{t_{0}}^{s} h(\tau) d \tau\right)\right) d s\right], t_{0} \leq t<b_{1},
$$

where $W, W^{-1}$ are the same functions as in Lemma 2.3 and

$$
\left.b_{1}=\sup \left\{t \geq t_{0}: W(c)+\int_{t_{0}}^{t}\left(f(s)+g(s) \int_{t_{0}}^{s} h(\tau) d \tau\right)\right) d s \in \operatorname{domW}^{-1}\right\} .
$$

Lemma 2.5. [4] Let $u, f, g, h \in C\left(\mathbb{R}^{+}\right), w \in C((0, \infty))$ and $w(u)$ be nondecreasing in $u$. Suppose that for some $c>0$,

$u(t) \leq c+\int_{t_{0}}^{t} f(s) w(u(s)) d s+\int_{t_{0}}^{t} g(s)\left(\int_{t_{0}}^{s} h(\tau) w(u(\tau)) d \tau\right) d s, 0 \leq t_{0} \leq t$.

Then

$$
u(t) \leq W^{-1}\left[W(c)+\int_{t_{0}}^{t}\left(f(s)+g(s) \int_{t_{0}}^{s} h(\tau)\right) d s\right], t_{0} \leq t<b_{1},
$$

where $W, W^{-1}$ are the same functions as in Lemma 2.3 and

$$
b_{1}=\sup \left\{t \geq t_{0}: W(c)+\int_{t_{0}}^{t}\left(f(s)+g(s) \int_{t_{0}}^{s} h(\tau)\right) d s \in \operatorname{domW}^{-1}\right\} .
$$

Lemma 2.6. [10] Let $u, p, q, w$, and $r \in C\left(\mathbb{R}^{+}\right)$and suppose that, for some $c \geq 0$, we have

$$
u(t) \leq c+\int_{t_{0}}^{t} p(s) \int_{t_{0}}^{s}\left[q(\tau) u(\tau)+w(\tau) \int_{t_{0}}^{\tau} r(a) u(a) d a\right] d \tau d s, t \geq t_{0} .
$$

Then

(2.8) $u(t) \leq c \exp \left(\int_{t_{0}}^{t} p(s) \int_{t_{0}}^{s}\left[q(\tau)+w(\tau) \int_{t_{0}}^{\tau} r(a) d a\right] d \tau d s\right), t \geq t_{0}$. 
Lemma 2.7. [12] Let $u, p, q, w, r \in C\left(\mathbb{R}^{+}\right), w \in C((0, \infty))$ and $w(u)$ be nondecreasing in $u$. Suppose that for some $c \geq 0$,

$u(t) \leq c+\int_{t_{0}}^{t}\left(p(s) \int_{t_{0}}^{s}\left(q(\tau) w(u(\tau))+v(\tau) \int_{t_{0}}^{\tau} r(a) u(a) d a\right) d \tau\right) d s, t \geq t_{0}$.

Then

$u(t) \leq W^{-1}\left[W(c)+\int_{t_{0}}^{t}\left(p(s) \int_{t_{0}}^{s}\left(q(\tau)+v(\tau) \int_{t_{0}}^{\tau} r(a) d a\right) d \tau\right) d s\right], t_{0} \leq t<b_{1}$,

where $W, W^{-1}$ are the same functions as in Lemma 2.3 and

$$
\begin{aligned}
b_{1}=\sup \left\{t \geq t_{0}: W(c)\right. & +\int_{t_{0}}^{t}\left(p(s) \int_{t_{0}}^{s}(q(\tau)\right. \\
& \left.\left.\left.+v(\tau) \int_{t_{0}}^{\tau} r(a) d a\right) d \tau\right) d s \in \mathrm{domW}^{-1}\right\} .
\end{aligned}
$$

Lemma 2.8. [14] Let $u(t), f(t)$, and $g(t)$ be real-valued nonnegative continuous functions defined on $\mathbb{R}^{+}$, for which the inequality

$$
u(t) \leq u_{0}+\int_{0}^{t} f(s) u(s) d s+\int_{0}^{t} f(s)\left(\int_{0}^{s} g(\tau) u(\tau) d \tau\right) d s, t \in \mathbb{R}^{+},
$$

holds, where $u_{0}$ is a nonnegative constant. Then,

$$
u(t) \leq u_{0}\left(1+\int_{0}^{t} f(s) \exp \left(\int_{0}^{s}(f(\tau)+g(\tau)) d \tau\right)\right) d s, t \in \mathbb{R}^{+} .
$$

LEMma 2.9. [5] Let the following condition hold for functions $u(t)$, $v(t) \in C\left(\mathbb{R}^{+}\right)$and $k(t, u, v) \in C\left(\mathbb{R}^{+} \times \mathbb{R}^{+} \times \mathbb{R}^{+}, \mathbb{R}^{+}\right)$:

$$
u(t)-\int_{t_{0}}^{t} k(s, u(s), T u(s)) d s \leq v(t)-\int_{t_{0}}^{t} k(s, v(s), T v(s)) d s,
$$

$t \geq t_{0} \geq 0$ and $k(t, u, v)$ is monotone nondecreasing in $u$ and $v$ for each fixed $t \geq 0$. If $u\left(t_{0}\right)<v\left(t_{0}\right)$, then $u(t)<v(t), t \geq t_{0} \geq 0$.

\section{Main results}

In this section, we investigate uniform Lipschitz and asymptotic stability for solutions of the nonlinear perturbed differential systems using integral inequalities. 
Theorem 3.1. Suppose that $x=0$ of (2.1) is ULS. Let the following condition hold for (2.2):

$$
\int_{t_{0}}^{t}|g(s, y(s), T y(s))| d s \leq W(t,|y|, T|y|), 0 \leq t_{0} \leq t,
$$

where $W(t, u, v) \in C\left(\mathbb{R}^{+} \times \mathbb{R}^{+} \times \mathbb{R}^{+}, \mathbb{R}^{+}\right)$is monotone nondecreasing in $u$ and $v$ for each fixed $t \geq 0$ with $W(t, 0,0)=0$. Assume that $u(t)$ is any solution of the scalar differential equation

$$
u^{\prime}(t)=K W(t, u, T u), u\left(t_{0}\right)=u_{0}>0, K \geq 1,
$$

existing on $\mathbb{R}^{+}$such that $m\left(t_{0}\right)<u\left(t_{0}\right)$. If $u=0$ of (3.1) is $U L S$, then $y=0$ of (2.2) is also ULS whenever $K\left|y_{0}\right|<u_{0}$.

Proof. Let $y(t)=y\left(t, t_{0}, y_{0}\right)$ be any solution of (2.2). By Lemma 2.2, we have

$$
|y(t)| \leq|x(t)|+\int_{t_{0}}^{t}|\Phi(t, s, y(s))| \int_{t_{0}}^{s}|g(\tau, y(\tau), T y(\tau))| d \tau d s,
$$

where $\Phi\left(t, t_{0}, y_{0}\right)$ is the fundemental matrix of (2.4). Since $x=0$ of $(2.1)$ is ULS, it is ULSV by Corollary 3.6[7]. Therefore there exist $K>0$ and $\delta>0$ such that $\left|\Phi\left(t, t_{0}, y_{0}\right)\right| \leq K$ for $t \geq t_{0} \geq 0$. Thus, by the assmption, we obtain

$$
\begin{aligned}
& |y(t)|-K \int_{t_{0}}^{t} W(s,|y(s)|, T|y(s)|) d s \\
& \leq K\left|y_{0}\right|<u_{0}=u(t)-K \int_{t_{0}}^{t} W(s, u(s), T u(s)) d s .
\end{aligned}
$$

Hence $|y(t)|<u(t)$ by Lemma 2.9. Since $u=0$ of (3.1) is ULS, it easily follows that $y=0$ of (2.2) is ULS.

Corollary 3.2. Suppose that $x=0$ of (2.1) is ULS. Consider the scalar differential equation

$$
u^{\prime}(t)=K W(t, u, T u)=K a(t)\left[u+\int_{t_{0}}^{t} k(s) u(s) d s\right],
$$

where $u_{0} \geq 1, K \geq 1$ and $a, k \in C\left(\mathbb{R}^{+}\right)$satisfy the conditions:

(a) $\int_{t_{0}}^{t}|g(s, y(s), T y(s))| d s \leq W(t,|y|, T|y|)$, where $\int_{t_{0}}^{t} g(s, y(s), T y(s)) d s$ is in (2.2),

(b) $M\left(t_{0}\right)=\left(1+K \int_{t_{0}}^{\infty} a(s) \exp \left(\int_{t_{0}}^{s}(K a(\tau)+k(\tau)) d \tau\right) d s\right)<\infty$ and $b_{1}=\infty$.

Then $y=0$ of (2.2) is ULS. 
Proof. Let $u(t)=u\left(t, t_{0}, u_{0}\right)$ be any solution of (3.2). Then, by Lemma 2.8 , we obtain

$|u(t)| \leq u_{0}\left(1+K \int_{t_{0}}^{t} a(s) \exp \left(\int_{t_{0}}^{s}(K a(\tau)+k(\tau)) d \tau\right) d s\right) \leq M\left(t_{0}\right)\left|u_{0}\right|$,

Hence $u=0$ of (3.2) is ULS. By Theorem 3.1, the solution $y=0$ of (2.2) is ULS .

REMARK 3.1. In corollary 3.2 , it is needed that $b_{1}=\infty$. The condition $W(\infty)=\infty$ is too strong and it represents situations which are not stable. For example, if $w(u)=u^{\alpha}$, then only $\alpha \leq 1$ satisfies $W(\infty)=\infty$ and $\alpha<1$ is not stable. See [17].

Corollary 3.3. Suppose that $x=0$ of (2.1)is ULS. Consider the scalar differential equation

$$
u^{\prime}(t)=K W(t, u, T u)=K a(t)\left[u+\int_{t_{0}}^{t} k(s) w(u(s)) d s\right],
$$

where $u_{0} \geq 1, K \geq 1, u, w \in C\left(\mathbb{R}^{+}\right), w(u)$ is nondecreasing in $u$ and $\frac{1}{v} w(u) \leq w\left(\frac{u}{v}\right)$ for some $v>0$, and $a, k \in C\left(\mathbb{R}^{+}\right)$satisfy the conditions:

(a) $\int_{t_{0}}^{t}|g(s, y(s), T y(s))| d s \leq W(t,|y|, T|y|)$, where $\int_{t_{0}}^{t} g(s, y(s), T y(s)) d s$ is in (2.2),

(b) $M\left(t_{0}\right)=W^{-1}\left[W\left(u_{0}\right)+\int_{t_{0}}^{\infty} k(s) d s\right] \cdot \exp \left(\int_{t_{0}}^{\infty} K a(s) d s\right)<\infty, b_{1}=$ $\infty$, and $a, k \in L_{1}\left(\mathbb{R}^{+}\right)$.

Then $y=0$ of $(2.2)$ is ULS.

Proof. Let $u(t)=u\left(t, t_{0}, u_{0}\right)$ be any solution of (3.3). Then, by Lemma 2.3, we have

$$
\begin{aligned}
|u(t)| & \leq W^{-1}\left[W\left(u_{0}\right)+\int_{t_{0}}^{t} k(s) d s\right] \cdot \exp \left(\int_{t_{0}}^{t} K a(s) d s\right) \\
& =M\left(t_{0}\right) \leq M\left(t_{0}\right)\left|u_{0}\right|,
\end{aligned}
$$

Hence $u=0$ of (3.3) is ULS, and so by Theorem 3.1, the solution $y=0$ of $(2.2)$ is ULS .

Corollary 3.4. Suppose that $x=0$ of (2.1) is ULS. Consider the scalar differential equation

$$
u^{\prime}(t)=K W(t, u, T u)=K\left[a(t) w(u(t))+b(t) \int_{t_{0}}^{t} k(s) u(s) d s\right],
$$

where $w \in C\left((0, \infty), w(u)\right.$ is nondecreasing on $u$ and $u \leq w(u), u_{0} \geq 1$, $K \geq 1$ and $a, b, k \in C\left(\mathbb{R}^{+}\right)$satisfy the conditions: 
(a) $\int_{t_{0}}^{t}|g(s, y(s), T y(s))| d s \leq W(t,|y|, T|y|)$, where $\int_{t_{0}}^{t} g(s, y(s), T y(s)) d s$ is in (2.2),

(b) $M\left(t_{0}\right)=W^{-1}\left[W\left(u_{0}\right)+K \int_{t_{0}}^{\infty}\left(a(s)+b(s) \int_{t_{0}}^{s} k(s) d s\right)\right]<\infty$, $b_{1}=\infty$, and $a, b, k \in L_{1}\left(\mathbb{R}^{+}\right)$.

Then $y=0$ of (2.2) is ULS.

Proof. Let $u(t)=u\left(t, t_{0}, u_{0}\right)$ be any solution of (3.4). Then, Lemma 2.4 , we obtain

$$
\begin{aligned}
|u(t)| & \leq W^{-1}\left[W\left(u_{0}\right)+K \int_{t_{0}}^{t}\left(a(s)+b(s) \int_{t_{0}}^{s} k(s) d s\right)\right] \\
& \leq M\left(t_{0}\right) \leq M\left(t_{0}\right)\left|u_{0}\right|
\end{aligned}
$$

Hence $u=0$ of (3.4) is ULS. This implies that the solution $y=0$ of (2.2) is ULS by Theorem 3.1.

Theorem 3.5. For the perturbed (2.2), we suppose that

$$
\int_{t_{0}}^{t}|g(s, y(s), T y(s))| d s \leq a(t) w(|y(t)|)+b(t) \int_{t_{0}}^{t} k(s)|y(s)| d s,
$$

where $a, b, k \in C\left(\mathbb{R}^{+}\right), a, b, k \in L_{1}\left(\mathbb{R}^{+}\right), w \in C((0, \infty)$, and $w(u)$ is nondecreasing in $u, u \leq w(u)$, and $\frac{1}{v} w(u) \leq w\left(\frac{u}{v}\right)$ for some $v>0$,

$$
M\left(t_{0}\right)=W^{-1}\left[W(K)+K \int_{t_{0}}^{\infty}\left(a(s)+b(s) \int_{t_{0}}^{s} k(\tau) d \tau\right) d s\right],
$$

where $M\left(t_{0}\right)<\infty$ and $b_{1}=\infty$. If the zero solution of (2.1) is ULSV, the zero solution of (2.2) is ULS.

Proof. Let $x(t)=x\left(t, t_{0}, y_{0}\right)$ and $y(t)=y\left(t, t_{0}, y_{0}\right)$ be solutions of (2.1) and (2.2), respectively. Since $x=0$ of (2.1) is ULSV, there exist $K>0$ and $\delta>0$ such that $\left|\Phi\left(t, t_{0}, y_{0}\right)\right| \leq K$ for $t \geq t_{0} \geq 0$. In view of Lemma 2.2 and (3.5), we obtain

$$
\begin{aligned}
|y(t)| \leq|x(t)| & +\int_{t_{0}}^{t}|\Phi(t, s, y(s))|\left|\int_{t_{0}}^{s} g(\tau, y(\tau), T y(\tau)) d \tau\right| d s \\
\leq K\left|y_{0}\right| & +\int_{t_{0}}^{t} K\left|y_{0}\right| a(s) w\left(\frac{|y(s)|}{\left|y_{0}\right|}\right) d s \\
& +\int_{t_{0}}^{t} K\left|y_{0}\right| b(s) \int_{t_{0}}^{s} k(\tau) \frac{|y(\tau)|}{\left|y_{0}\right|} d \tau d s .
\end{aligned}
$$

Set $u(t)=|y(t)|\left|y_{0}\right|^{-1}$. Then, by Lemma 2.4, we have 


$$
|y(t)| \leq\left|y_{0}\right| W^{-1}\left[W(K)+K \int_{t_{0}}^{t}\left(a(s)+b(s) \int_{t_{0}}^{s} k(\tau) d \tau\right) d s\right] .
$$

The above estimation yields the desired result. Hence the proof is complete.

TheOREm 3.6. For the perturbed (2.2), we suppose that

$$
|g(t, y(t), T y(t))| \leq a(t) w(|y(t)|)+b(t) \int_{t_{0}}^{t} k(s)|y(s)| d s,
$$

where $a, b, k \in C\left(\mathbb{R}^{+}\right), a, b, k \in L_{1}\left(\mathbb{R}^{+}\right), w \in C((0, \infty)$, and $w(u)$ is nondecreasing in $u, u \leq w(u)$, and $\frac{1}{v} w(u) \leq w\left(\frac{u}{v}\right)$ for some $v>0$,

$$
M\left(t_{0}\right)=W^{-1}\left[W(K)+K \int_{t_{0}}^{\infty} \int_{t_{0}}^{s}\left(a(\tau)+b(\tau) \int_{t_{0}}^{\tau} k(r) d r\right) d \tau d s\right]
$$

where $M\left(t_{0}\right)<\infty$ and $b_{1}=\infty$. If the zero solution of (2.1) is $U L S V$, the zero solution of (2.2) is ULS.

Proof. Let $x(t)=x\left(t, t_{0}, y_{0}\right)$ and $y(t)=y\left(t, t_{0}, y_{0}\right)$ be solutions of (2.1) and (2.2), respectively. Using Lemma 2.2, ULSV condition of $x=0$ of (2.1), and (3.7), we obtain

$$
\begin{aligned}
|y(t)| \leq & |x(t)|+\int_{t_{0}}^{t}|\Phi(t, s, y(s))| \int_{t_{0}}^{s}|g(\tau, y(\tau), T y(\tau))| d \tau d s \\
\leq & K\left|y_{0}\right|+\int_{t_{0}}^{t} K\left|y_{0}\right| \int_{t_{0}}^{s}\left[a(\tau) w\left(\frac{|y(\tau)|}{\left|y_{0}\right|}\right) d \tau d s\right. \\
& \left.+\int_{t_{0}}^{t} K\left|y_{0}\right| \int_{t_{0}}^{s} b(\tau) \int_{t_{0}}^{\tau} k(r) \frac{|y(r)|}{\left|y_{0}\right|} d r d \tau\right] d s .
\end{aligned}
$$

Set $u(t)=|y(t)|\left|y_{0}\right|^{-1}$. Then, an application of Lemma 2.7 yields

$$
|y(t)| \leq\left|y_{0}\right| W^{-1}\left[W(K)+K \int_{t_{0}}^{t} \int_{t_{0}}^{s}\left(a(\tau)+b(\tau) \int_{t_{0}}^{\tau} k(r) d r\right) d \tau d s\right] .
$$

Thus we have $|y(t)| \leq M\left(t_{0}\right)\left|y_{0}\right|$ for some $M\left(t_{0}\right)>0$ whenever $\left|y_{0}\right|<\delta$. This completes the proof. 
Theorem 3.7. Let the solution $x=0$ of (2.1) be EASV. Assume that the perturbing term $\int_{t_{0}}^{t} g(s, y(s), T y(s)) d s$ satisfies

$$
\begin{aligned}
& \int_{t_{0}}^{t}|g(s, y(s), T y(s))| d s \\
& \leq e^{-\alpha t}\left(a(t) w(|y(t)|)+b(t) \int_{t_{0}}^{t} k(s) w(|y(s)|) d s\right),
\end{aligned}
$$

where $\alpha>0, a, b, k, w \in C\left(\mathbb{R}^{+}\right), a, b, k \in L_{1}\left(\mathbb{R}^{+}\right)$and $w(u)$ is nondecreasing in $u$. If

$$
M\left(t_{0}\right)=W^{-1}\left[W(c)+K \int_{t_{0}}^{\infty}\left(a(s)+b(s) \int_{t_{0}}^{s} k(\tau) d \tau\right) d s\right]<\infty, b_{1}=\infty
$$

where $c=K\left|y_{0}\right| e^{\alpha t_{0}}$, then all solutions of (2.2) approch zero as $t \rightarrow \infty$

Proof. Let $x(t)=x\left(t, t_{0}, y_{0}\right)$ and $y(t)=y\left(t, t_{0}, y_{0}\right)$ be solutions of (2.1) and (2.2), respectively. Using Lemma 2.2 and (3.9), we obtain

$$
\begin{aligned}
|y(t)| \leq & |x(t)|+\int_{t_{0}}^{t}|\Phi(t, s, y(s))|\left|\int_{t_{0}}^{s} g(\tau, y(\tau), T y(\tau)) d \tau\right| d s \\
\leq & K\left|y_{0}\right| e^{-\alpha\left(t-t_{0}\right)}+\int_{t_{0}}^{t} K e^{-\alpha(t-s)}\left[e^{-\alpha s} a(s) w(|y(s)|)\right. \\
& \left.+K b(s) e^{-\alpha s} \int_{t_{0}}^{s} k(\tau) w(|y(\tau)|) d \tau\right] d s .
\end{aligned}
$$

Set $u(t)=|y(t)| e^{\alpha t}$. Then, since $w(u)$ is nondecreasing, by Lemma 2.5 we obtain

$$
|y(t)| \leq e^{-\alpha t} W^{-1}\left[W(c)+K \int_{t_{0}}^{t}\left(a(s)+b(s) \int_{t_{0}}^{s} k(\tau) d \tau\right) d s\right], t \geq t_{0} \geq 0,
$$

where $c=K\left|y_{0}\right| e^{\alpha t_{0}}$. The above estimation yields the desired result.

Theorem 3.8. Let the solution $x=0$ of (2.1) be EASV. Assume that the perturbing term $\int_{t_{0}}^{t} g(s, y(s), T y(s)) d s$ satisfies

$$
|g(t, y(t), T y(t))| \leq e^{-\alpha t}\left(a(t)|y(t)|+b(t) \int_{t_{0}}^{t} k(s)|y(s)| d s\right),
$$

where $\alpha>0, a, b, k \in C\left(\mathbb{R}^{+}\right), a, b, k \in L_{1}\left(\mathbb{R}^{+}\right), w(u)$ is nondecreasing in $u$, and $\frac{1}{v} w(u) \leq w\left(\frac{u}{v}\right)$ for some $v>0$. If 


$$
\begin{aligned}
M\left(t_{0}\right)= & c \exp \left(\int_{t_{0}}^{\infty} K e^{\alpha s} \int_{t_{0}}^{s} e^{-\alpha \tau}[a(\tau)\right. \\
& \left.\left.+b(\tau) \int_{t_{0}}^{\tau} k(r) d r\right] d \tau d s\right)<\infty, t \geq t_{0} \geq 0 .
\end{aligned}
$$

where $c=K\left|y_{0}\right| e^{\alpha t_{0}}$, then all solutions of (2.2) approch zero as $t \rightarrow \infty$

Proof. Let $x(t)=x\left(t, t_{0}, y_{0}\right)$ and $y(t)=y\left(t, t_{0}, y_{0}\right)$ be solutions of (2.1) and (2.2), respectively. By the assumption, we have $\left|\Phi\left(t, t_{0}, y_{0}\right)\right| \leq$ $K e^{-c\left(t-t_{0}\right)}$ for some $K>0$ and $c>0$. Using Lemma 2.2 and (3.11), we obtain

$$
\begin{aligned}
|y(t)| \leq & |x(t)|+\int_{t_{0}}^{t}|\Phi(t, s, y(s))| \int_{t_{0}}^{s}|g(\tau, y(\tau), T y(\tau))| d \tau d s \\
\leq & K\left|y_{0}\right| e^{-\alpha\left(t-t_{0}\right)}+\int_{t_{0}}^{t} K e^{-\alpha(t-s)} \int_{t_{0}}^{s} e^{-\alpha \tau}[a(\tau)|y(\tau)| \\
& \left.+b(\tau) \int_{t_{0}}^{\tau} k(r)|y(r)| d r\right] d \tau d s .
\end{aligned}
$$

Set $u(t)=|y(t)| e^{\alpha t}$. Then, since $e^{\alpha t} \geq 1$, an application of Lemma 2.6 obtains

$$
\begin{aligned}
|y(t)| & \leq c e^{-\alpha t} \exp \left(\int_{t_{0}}^{t} K e^{\alpha s} \int_{t_{0}}^{s} e^{-\alpha \tau}\left[a(\tau)+b(\tau) \int_{t_{0}}^{\tau} k(r) d r\right] d \tau d s\right) \\
& \leq c e^{-\alpha t} M\left(t_{0}\right), t \geq t_{0} \geq 0,
\end{aligned}
$$

where $c=K\left|y_{0}\right| e^{\alpha t_{0}}$. From the above estimation, we obtain the desired result.

\section{Acknowledgements}

The author is very grateful for the referee's valuable comments.

\section{References}

[1] V. M. Alekseev, An estimate for the perturbations of the solutions of ordinary differential equations, Vestn. Mosk. Univ. Ser. I. Math. Mekh. 2 (1961), 2836(Russian).

[2] F. Brauer and A. Strauss, Perturbation of nonlinear systems of differential equations, III, J. Math. Anal. Appl. 31 (1970), 37-48.

[3] F. Brauer, Perturbation of nonlinear systems of differential equations, IV, J. Math. Anal. Appl. 37 (1972), 214-222. 
[4] S. K. Choi and N. J. Koo, h-stability for nonlinear perturbed systems, Ann. of Diff. Eqs. 11 (1995), 1-9.

[5] S. K. Choi, Y. H. Goo, and N. J. Koo, Lipschitz and exponential asymptotic stability for nonlinear functional systems, Dynamic Systems and Applications 6 (1997), 397-410.

[6] S. K. Choi, N. J. Koo, and S. M. Song, Lipschitz stability for nonlinear functional differential systems, Far East J. Math. Sci(FJMS)I 5 (1999), 689-708.

[7] F. M. Dannan and S. Elaydi, Lipschitz stability of nonlinear systems of differential systems, J. Math. Anal. Appl. 113 (1986), 562-577.

[8] S. Elaydi and H. R. Farran, Exponentially asymptotically stable dynamical systems, Appl. Appl. 25 (1987), 243-252.

[9] P. Gonzalez and M. Pinto, Stability properties of the solutions of the nonlinear functional differential systems, J. Math. Appl. 181 (1994), 562-573.

[10] Y. H. Goo and S. B. Yang, h-stability of the nonlinear perturbed differential systems via $t_{\infty}$-similarity, J. Chungcheong Math. Soc. 24 (2011), 695-702.

[11] Y. H. Goo and S. B. Yang, h-stability of nonlinear perturbed differential systems via $t_{\infty}$-similarity, J. Korean Soc. Math. Educ. Ser. B: Pure Appl. Math. 19 (2012), 171-177.

[12] Y. H. Goo, Boundedness in the perturbed differential systems, J. Korean Soc. Math. Educ. Ser. B: Pure Appl. Math. 20 (2013), 223-232.

[13] V. Lakshmikantham and S. Leela, Differential and Integral Inequalities: Theory and Applications Vol., Academic Press, New York and London, 1969.

[14] B. G. Pachpatte, A note on Gronwall-Bellman inequality, J. Math. Anal. Appl. 44 (1973), 758-762.

[15] M. Pinto, Perturbations of asymptotically stable differential systems, Analysis 4 (1984), 161-175.

[16] M. Pinto, Integral inequalities of Bihari-type and applications, Funkcial. Ekvac. 33 (1990), 387-404.

[17] M. Pinto, Variationally stable differential system, J. Math. Anal. Appl. 151 (1990), 254-260.

Department of Mathematics

Hanseo University

Chungnam 356-706, Republic of Korea

E-mail: yhgoo@hanseo.ac.kr

**

Institute of Business and Economic Research

Harbin University of Commerce

Harbin 150076, P. R. China

E-mail: yinhua_j@hotmail.com 